\title{
Path-length-resolved dynamic light scattering: modeling the transition from single to diffusive scattering
}

\author{
Adam Wax, Changhuei Yang, Ramachandra R. Dasari, and Michael S. Feld
}

\begin{abstract}
Dynamic light-scattering spectroscopy is used to study Brownian motion within highly scattering samples. The fluctuations of the light field that is backscattered by a suspension of polystyrene microspheres are measured as power spectra by use of low-coherence interferometry to obtain path-length resolution. The data are modeled as the sum of contributions to the detected light weighted by a Poisson probability for the number of events that each component has experienced. By analyzing the broadening of the power spectra as a function of the path length for various sizes of particles, we determine the contribution of multiple scattering to the detected signal as a function of scattering anisotropy. (C) 2001 Optical Society of America
\end{abstract}

OCIS codes: $\quad 290.4210,290.7050,030.5290,120.3180$.

\section{Introduction}

In dynamic light scattering (DLS) one examines the intensity fluctuations of a scattered light field to determine the structure and the dynamics of samples that weakly scatter light.1,2 Multiply scattered light in DLS measurements is often viewed as a source of noise and reduces the effectiveness of DLS in thick turbid samples. Conversely, in diffusing-wave spectroscopy (DWS), one uses intensity fluctuations to determine the properties of thick turbid samples, ${ }^{3,4}$ but the DWS theory begins to break down for optically thin samples. ${ }^{5,6}$

The regime between these two extremes, when light has been scattered only a few times, is not well understood. Improving the understanding of the detection of low-order scattered light not only would aid in photon-correlation experiments such as those performed with DLS and DWS but also is relevant for confocal microscopy ${ }^{7}$ and optical coherence tomography, ${ }^{8}$ in which contrast and image quality are known to be degraded by multiple scattering.

Recently a new instrument was developed that permits measurements of the dynamics of singly and

The authors are with the G. R. Harrison Spectroscopy Laboratory, Massachusetts Institute of Technology, 77 Massachusetts Avenue, Cambridge, Massachusetts 02139. A. Wax's e-mail address is awax@mit.edu.

Received 29 August 2000; revised manuscript received 19 March 2001.

0003-6935/01/244222-06\$15.00/0

(C) 2001 Optical Society of America low-order scattered light in a thick turbid sample by exploiting the path-length resolution obtained in lowcoherence interferometry. ${ }^{9,10}$ Studies with this instrument showed that the detection of multiply scattered light as a function of the path length within the scattering medium depends both on the geometry of the detection optics and on the anisotropy of the scattering. These studies modeled the singlescattering and the diffusive regimes of light fluctuations but did not model the transition between the two. 9,10

In this paper, we present DLS data that was measured with low-coherence interferometry. The data were obtained as power spectra and then individually fitted to Lorentzian distributions. We were then able to study the width and the amplitude of the power spectrum as a function of the photon path length within a scattering medium. We used a wellcharacterized dynamic system, a suspension of polystyrene microspheres in water undergoing Brownian motion, to study the detection of multiply scattered light. By comparing the trends in the measured power spectra for various-sized microspheres with a theoretical treatment that decomposes the total power spectrum by the number of scattering events, we were able to correlate the detection of multiply scattered light with scattering anisotropy.

The experimental setup is shown in Fig. 1. The light source is a superluminescent diode from EG\&G with a center wavelength of $845 \mathrm{~nm}$ and a FWHM bandwidth of $22 \mathrm{~nm}$. The calculated coherence length of $14.3 \mu \mathrm{m}$ for this source agrees well with the experimentally measured coherence length of 13.6 


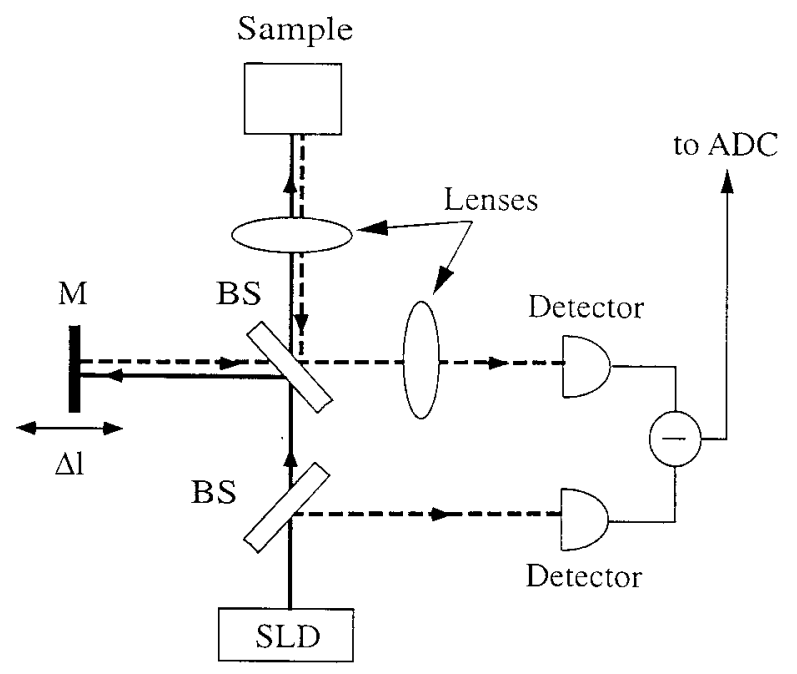

Fig. 1. Schematic of the experimental setup: BSs, beam splitters; M, mirror; ADC, analog-to-digital converter; SLD, superluminescent diode.

$\mu \mathrm{m}$ that we obtained by replacing the sample with a mirror and scanning the reference arm to generate a heterodyne signal. For the DLS studies, the reference mirror is fixed in position, and a heterodyne signal is generated as a result of Doppler shifts that are imparted to the light after scattering by moving particles within the sample. The coherence gate selects the path length of the light within the medium for detection. When the reference mirror is displaced by a distance $\Delta z$, light is detected that has traveled a distance of $L=2 \Delta z / n_{0}$ within the medium, where $n_{0}$ is the refractive index of the background medium (in our case, $n_{0}=1.33$ for water).

The lenses used in the low-coherence interferometry setup are 10-cm focal-length achromats arranged in a conventional $4 f$ imaging system ${ }^{11}$ such that the phase and the amplitude of the signal's electric field are both reproduced in the detector plane. It is essential that the $4 f$ system be used for examining multiple scattering with heterodyne detection because spatially varying phase shifts that are due to propagation can skew the results of field-mixing experiments. The two photodetectors are components of a New Focus Model 2007 autobalanced photoreceiver. This receiver provides two advantages: The dc level is subtracted from the signal photocurrent, and the balanced detectors allow for cancellation of any power fluctuations in the light source, permitting the light field's fluctuations that are due to the sample to be studied free from artifacts. The ac photocurrent is digitized with a $333-\mathrm{kHz}$ analog-to-digital converter. The digitized signal is Fourier transformed and its magnitude squared to yield the power spectrum of the signal.

The contribution of the time-varying scattered electric field to the detected ac photocurrent can be written as the sum of two components: The first component is due to the correlation of the scattered signal field with itself, $I_{\mathrm{SELF}} \sim\left\langle E_{S} E_{S} *\right\rangle$. The second is due to the interference between the scattered signal field and the time-invariant reference field, $I_{\mathrm{BEAT}}$ $\sim\left\langle E_{S} E_{R}{ }^{*}\right\rangle$. The self-term is an interesting feature of these experiments that was unaccounted for in previous dynamics experiments that used lowcoherence light.9,10 In the present experiments it is regarded as a noise term and is accounted for in our model, as is the mechanical noise that is not removed by the balanced detection. The baseline noise is measured by selection of the coherence gate so that it detects light with a path length that is too short for the light to have interacted with the medium, effectively setting the beat term to zero. The power spectrum of this baseline noise is calculated and subtracted from the calculated power spectrum of the total photocurrent obtained during the experiment, yielding the power spectrum of only the beat term. Because the reference field does not vary over time, the power spectrum of the beat term is just the Fourier transform of the signal field's autocorrelation function. This procedure contrasts with intensity autocorrelation measurements in which one takes the square root to obtain the field autocorrelation by using the Siegert relation. ${ }^{1}$

The power spectrum of light that is scattered by a monodisperse suspension of particles undergoing Brownian motion and is then heterodyne detected is a Lorentzian distribution ${ }^{1,2}$ :

$$
S(f)=\frac{1}{f_{0}} \frac{A}{1+\left(f / f_{0}\right)^{2}},
$$

where $A$ is the amplitude and $f_{0}$ is the width at halfmaximum of the distribution. For single-scattered light the power spectrum's linewidth can be related by

$$
\pi f_{0}=2 k^{2} D_{B}
$$

to the diffusion constant of the Brownian motion

$$
D_{B}=\kappa_{B} T / 3 \pi \eta a,
$$

where $k$ is the optical wave vector, $\kappa_{B}$ is the Boltzmann constant, $T$ is the temperature, $\eta$ is the viscosity of the background liquid $[\eta=1.0$ counts/s (cps) for water], and $a$ is the diameter of the particle. For diffusive scattering, the power spectrum is still Lorentzian, but the linewidth now depends on the photon path length in the medium $L$ and is given by ${ }^{12}$

$$
\pi f_{0}=k^{2} D_{B} \frac{L}{l}(1-g),
$$

where $l$ is the photon mean free scattering path and $g=\langle\cos \theta\rangle$ is the scattering anisotropy.

One can derive the power spectrum for multiple scattering by considering the velocity shifts that are imparted to a photon as it travels through the scattering medium. At each scattering event the photon encounters a particle in Brownian motion; i.e., its velocity has a thermal distribution

$$
F(v) \propto \exp \left(-v^{2} / v_{\mathrm{th}}{ }^{2}\right) .
$$


For $n$ scattering events the net velocity shift imparted to the photon is given by a convolution of $n$ of these Gaussian distributions, yielding

$$
F_{n}(v) \propto \exp \left(-v^{2} / n v_{\mathrm{th}}{ }^{2}\right) .
$$

As the thermal velocity is represented in the Brownian diffusion constant through the temperature [Eq. (2)],

$$
D_{B} \propto k_{B} T \propto v_{\text {th }}^{2},
$$

we can include the effects of multiple scattering by simply multiplying $D_{B}$ by the number of scattering events $n$. Thus we can write the power spectrum for light that has scattered $n$ times as

$$
S_{n}(f)=\frac{1}{n f_{0}} \frac{A}{1+\left(f / n f_{0}\right)^{2}},
$$

where $f_{0}$ is the linewidth for single scattering, as given by Eq. (3).

In our experiments, we examine the effects of the photon path length in the scattering medium on the power spectrum. Although selecting a particular path length for detection does not directly specify the number of scattering events, one can use a randomwalk treatment to determine the probability of $n$ scattering events for a given path length $L$. The probability is given by a Poisson distribution

$$
P_{n}(\alpha, L)=\exp (-\alpha L / l) \frac{(\alpha L / l)^{n}}{n !},
$$

where $l$ is the scattering mean free path and $\alpha$ is an empirical parameter that represents a decreased probability of photon detection after $n$ scattering events that is due to various factors such as the geometry of the scattering experiment, the anisotropy of the scattering, and boundary reflections. From Eq. (9), we can see that the mean number of scattering events is given as $\alpha L / l$, indicating that the detected photons have a longer mean free path (when $\alpha<1$ ) than that given by the scattering mean free path $l$. The scaling of $\alpha$ is discussed below.

Using Eqs. (8) and (9), we can write the total power spectrum of fluctuations by summing over all possible numbers of scattering events, which yields

$$
S_{T}(f, \alpha, L)=\exp (-\beta L / l) \sum_{n=1}^{\infty} P_{n}(\alpha, L) S_{n}(f) .
$$

In Eq. (10) an empirical exponential attenuation factor $[\exp (-\beta L / l)]$ was added to account for the changes in amplitude seen in our data. Its origin and significance are discussed below.

Although it was derived independently, Eq. (10) is similar in form and origin to an expression derived by Bonner and Nossal to describe Doppler measurements of blood flow in tissue. ${ }^{13}$ However, there are two key differences between the two forms: First, Bonner and Nossal ${ }^{13}$ cast their expression in terms of a mean scattering path length instead of the actual path length, as given in Eq. (10). Second, they re- quire a more complicated form for $S_{n}$, the power spectrum of light for $n$ scatterings, than that shown in Eq. (8). These differences are not surprising considering that the two forms are intended to describe different problems. The Bonner-Nossal form could easily be adapted to describe our measurements.

There are several advantages to our model that one can recognize by comparing it with the established theory of DWS. In DWS theory the field's autocorrelation function, $g(\tau)=\left\langle E(t) E^{*}(t+\tau)\right\rangle$, is calculated from the equation ${ }^{6}$

$$
g(\tau)=\int_{0}^{\infty} P(S) \exp (-x S / 3) \mathrm{d} S,
$$

where $x=2 \pi f_{0} \tau$ and $P(S)$ is the probability density for a particle to have traveled a path of length $S=$ $L(1-g) / l$. The parameters $f_{0}, L, g$, and $l$ are as given above for Eq. (4) for particles with Brownian dynamics. Fourier-transforming Eq. (11) with respect to $\tau$ yields the power spectrum $S(f)$ of the field fluctuations

$$
S(f)=\int_{0}^{\infty} P(S) S\left(f, f_{0}\right) \mathrm{d} S,
$$

where $S\left(f, f_{0}\right)$ is given in Eq. (1) and $f_{0}$ is given in Eq. (4).

Aside from the obvious difference between our treatment [Eq. (10)], which examines only a particular photon path length, and DWS theory [Eq. (12)], which is integrated over all possible photon path lengths, there are other distinctions that merit discussion: First, to evaluate an analytical form requires that DWS theory approximates the net change in the momentum of the discrete scattering events by averaging over the scattering form factor. The use of this approximation allows Eq. (11) to be written as an integral over photon paths. This continuum approximation is valid in the limit of a large number of scattering events but fails for thin samples or short path lengths for which only a few scattering events take place.

To find an analytical form, one must make an additional approximation: $P(S)$ is found by use of a diffusion approximation that describes the photon propagation. In contrast to DWS theory, our discrete-summation form can be evaluated analytically, provided that the power spectrum for any of $n$ scattering events is a Lorentzian distribution, which is a valid assumption for Brownian motion that may not be generally true for all types of motion. In addition, our theory includes factors such as anisotropy, boundary reflections, and the experiment geometry by use of the phenomenological factor $\alpha$, whereas DWS theory incorporates these factors by the alteration of the form of $P(S)$. Finally, a comparison of our theory with DWS theory reveals that, by using low-coherence interferometry to probe the dynamics of a sample undergoing Brownian motion, one can 
Table 1. Properties of Microspheres

\begin{tabular}{lcccccccc}
\hline & \multicolumn{5}{c}{ Diameter of Microsphere (nm) } \\
\cline { 2 - 7 } \multicolumn{1}{c}{ Property } & 110 & 162 & 202 & 258 & 356 & 426 & 535 \\
\hline g Factor (Mie theory) & 0.05 & 0.112 & 0.175 & 0.289 & 0.557 & 0.689 \\
Density (particles $/ \mu \mathrm{m}^{3}$ ) & 143.5 & 19.5 & 6.14 & 1.66 & 0.381 & 0.159 & 0.742 \\
Volume fraction $(\%)$ & 10 & 4.3 & 2.65 & 1.5 & 0.9 & 0.64 \\
\hline
\end{tabular}

obtain the probability density $P(S)$ for a given experimental geometry and scattering anisotropy.

In the experiments power spectra are measured as a function of path length for polystyrene microspheres of several diameters $a$ that range from 110 to $535 \mathrm{~nm}$. The properties of the microspheres are summarized in Table 1. For all sizes of spheres the concentration was chosen to yield a scattering mean free path of $156 \mu \mathrm{m}$ from Mie theory except for the 110-nm microspheres for which only a maximum mean free path of $196 \mu \mathrm{m}$ could be obtained by use of stock solutions.

The measured power spectrum at each photon path length was fitted by a least-squares fit. Figure 2 shows the typical power spectra measured in our experiments for 258-nm microspheres at two photon path lengths, as indicated. The line shape is Lorentzian and can be characterized completely by its amplitude $A$ and its width $f_{0}$, as given in Eq. (1), although the spectra shown in Fig. 2 were normalized for display purposes.

Figure 3 shows typical data for the fitted amplitude and linewidth as functions of the photon path length within the medium for 258-nm microspheres. Figure 3(a) shows the fitted linewidth of the power spectrum versus the path length compared with the predicted width that is due to single scattering [Eq. (3)] and with the width predicted by diffusion theory [Eq. (4)]. Figure 3(b) shows the same fitted linewidths and the linewidths predicted by the theory presented above [Eq. (10)]. Figure 3(c) shows the amplitude of the power spectrum as a function of the path delay, which is also compared with the theoretical prediction from Eq. (10).

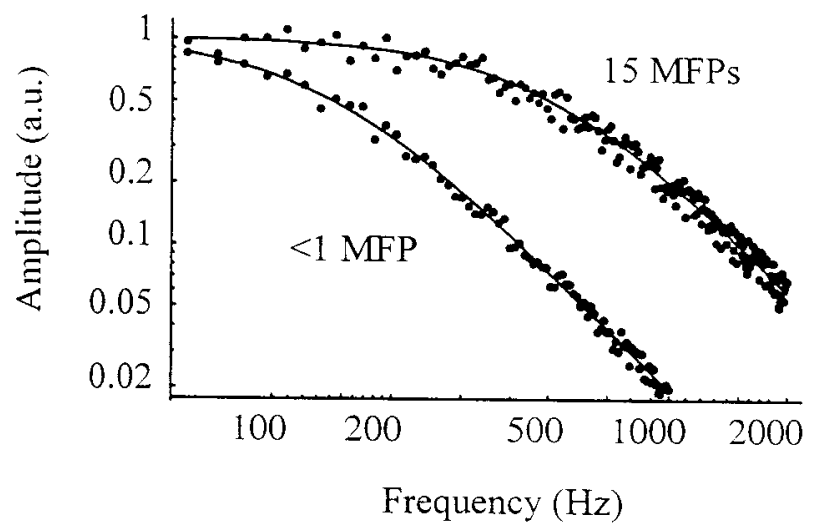

Fig. 2. Typical frequency spectra for 258-nm microspheres at the photon path lengths indicated. MFPs, mean free paths.
The data shown in Fig. 3 are typical for microsphere sizes of 202-535 nm. Their notable features are that the power spectra in this regime of scatterer sizes have linewidths that agree well with single-
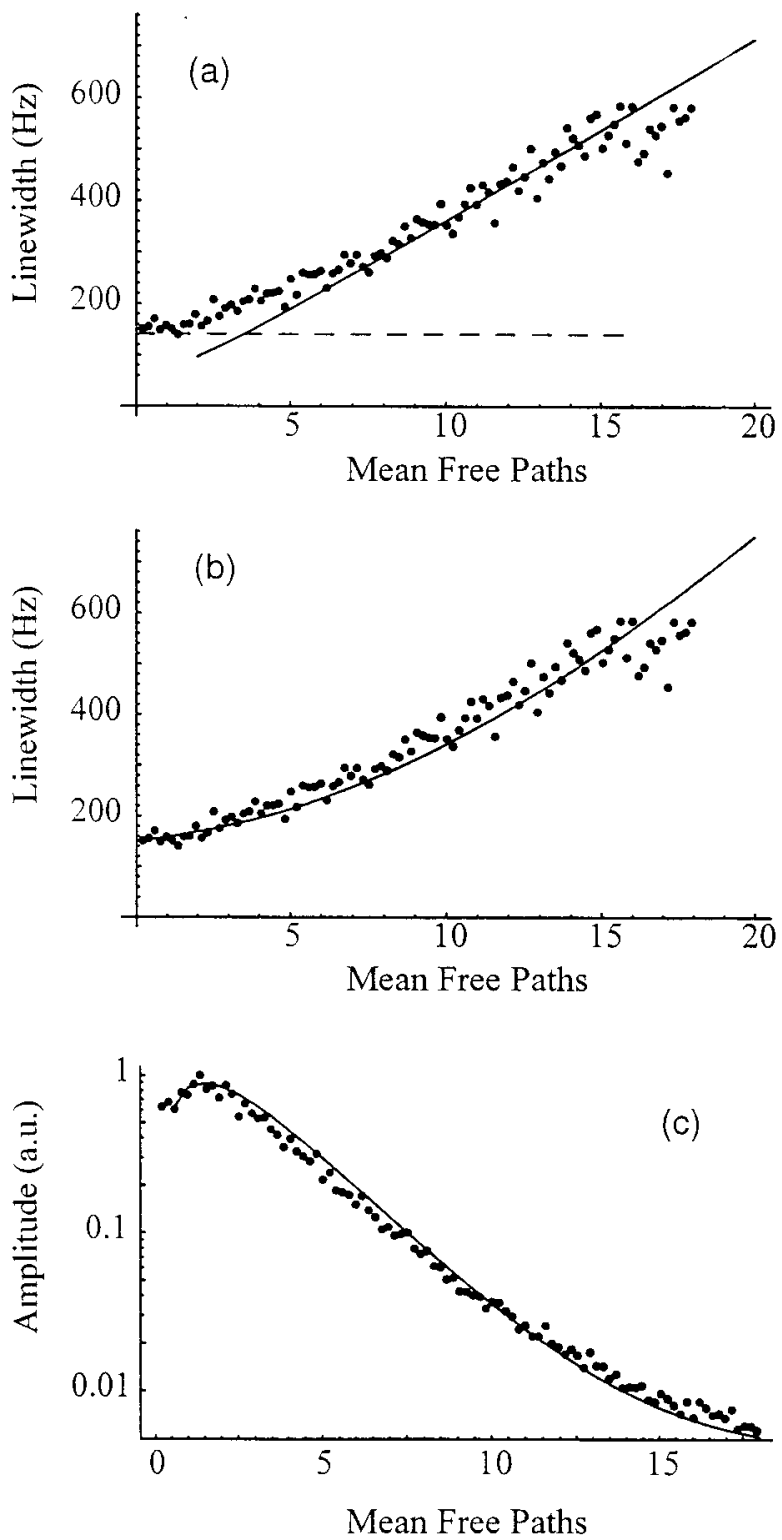

Fig. 3. (a), (b) Fitted linewidths and (c) amplitudes of the Lorentzian power spectrum measured as a function of the photon path length for 258-nm microspheres. Data (points) are shown compared with (a) single-scattering (dashed line) and diffusion-theory (solid line) predictions and (b), (c) the theoretical predictions (solid curves) presented in this paper. 

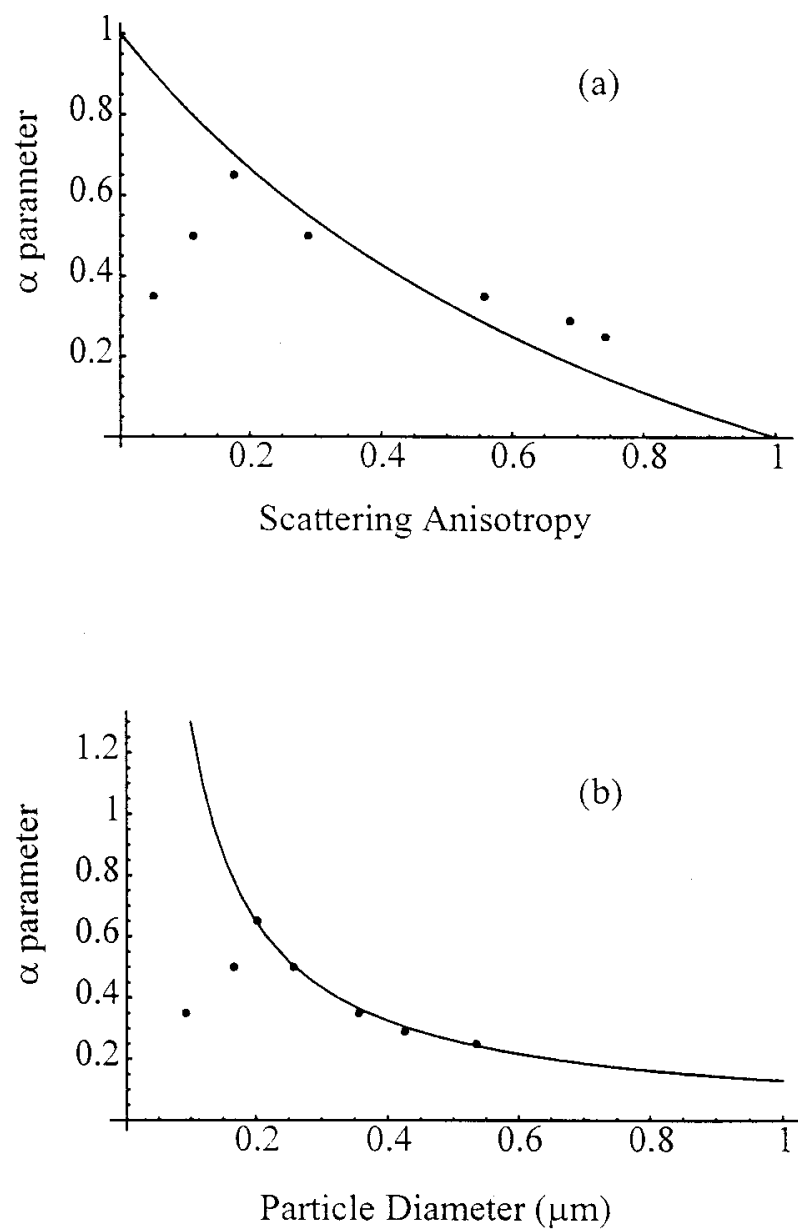

Fig. 4. Trends in the empirical parameter $\alpha$ compared with (a) scattering anisotropy and (b) particle diameter. The points represent the measured data; the solid curves represent the theory.

scattering theory predictions for short path lengths and with those of diffusion theory for longer path lengths. Our theoretical treatment can predict the behavior of the amplitudes and linewidths of the power spectra in this regime across the full range of path lengths from single scattering through diffusive transport.

To get good agreement between our theory and the data, we employed the $\alpha$ parameter introduced in Eq. (9). This parameter is included to account for the fact that a photon has a reduced probability of returning to the surface for detection the more scattering events it experiences. The notion of scaling the number of scattering events was introduced by Gandjbakhche et al. ${ }^{14}$ to describe anisotropic random walks. They found by using Monte Carlo calculations that they could include scattering anisotropy in the probability of a photon's experiencing $n$ scattering events and then returning to the surface by scaling the number of scattering events as $n(1-g) /(1+g)$. This result would suggest that our $\alpha$ parameter should scale in the same way.

Figure 4(a) shows a comparison of the $\alpha$ parameter that we obtained by fitting our theory to the data of the relation given by Gandjbakhche et al. ${ }^{14}$ We can see that their form agrees fairly well for particle sizes in the 200-535-nm regime. From Fig. 4(b), we can see that the parameter $\alpha$ is better fitted in this regime by a function that is inversely proportional to the particle diameter. The excellent agreement between theory and measurement in this regime suggests that there is some physical basis for the relation between particle size and the reduced probability for a photon to return to the surface.

We employ a second empirical parameter to obtain good agreement between the power spectrum's amplitude in our theory and in the data, an exponential decay factor: $\exp (-\beta L / l)$. For simple transmission through turbid media, one would expect this factor to be the ordinary exponential decay $\exp (-L /$ $l$ ); however, because the power spectrum's amplitude that was measured in our experiments is for light that followed a highly constrained path, confined in time, space, and momentum, it is not surprising that this factor is somewhat modified. As is shown by Yao and Wang ${ }^{15}$ in Monte Carlo simulations, the attenuation coefficient can depend strongly on the detection geometry and the properties of the medium. It is interesting that we find this factor to be constant $(\beta \sim 0.67)$ for all the diameters of microspheres used in the experiments. This result indicates that the medium-dependent part of the signal, i.e., the part that depends on particle size, scattering anisotropy, and the number of scattering events, is incorporated entirely into the $\alpha$ parameter, whereas the $\beta$ parameter represents the medium-independent part, i.e., boundary reflections and experimental geometry, because it depends on only the penetration depth.

Examining the trends of the $\alpha$ parameter shown in Fig. 4 makes it evident that the 110- and the 162-nm microspheres exhibit different scattering characteristics from the larger particles that we studied. For these two sizes, we found that, for short photon path lengths, we correctly measured the linewidth that was predicted as a result of single scattering but that, for longer photon path lengths, the diffusion theory does not correctly describe the growth of the linewidth. We can use our theory to model the behavior of the linewidth and the amplitude as functions of path length, but the required parameter $\alpha$ does not appear to be consistent with the values obtained for the larger particles.

The unusual behavior seen for the two smallerdiameter particles is most likely due to interparticle correlations that arise from the high volume fraction $\phi$ needed for the short mean free path. The 110-nm particle suspension has a value of $\phi=0.10$, and the $162-\mathrm{nm}$ suspension has a value of $\phi=0.044$. Previous studies ${ }^{12,16}$ showed that the transport mean free path predicted by Mie theory, $l^{*}=l /(1-g)$, is not valid for high volume fractions. In those studies it was shown that interparticle correlations skew $l^{*}$ to a higher value than that predicted by Mie theory, suggesting that the scattering anisotropy $g$ is larger than predicted. This result is consistent with our findings that the diffusion-theory prediction based on Mie theory does not describe the linewidth change for 
the smaller spheres and that the $\alpha$ parameter that we obtained for them would be more appropriate for larger spheres with larger $g$ factors. Improvements in the signal-to-noise ratio of our system through the use of quieter more-specialized amplifiers would permit studies of more-dilute samples of microspheres and thus enable further studies of the effect of the volume fraction on DLS to be conducted.

In conclusion, we have applied path-lengthresolved DLS spectroscopy to suspensions that are undergoing Brownian motion. We have been able to describe the changes in amplitude and linewidth of the measured power spectrum of scattered-light fluctuations by using a theory that dissects the scattering on the basis of the number of scattering events. By examining these changes as a function of particle size, we have been able to compare the scaling of the number of scattering events with scatterer anisotropy. We have found a reasonable agreement with existing theory and have suggested an alternative scaling relation. Further studies will apply our methods to biological tissues in an effort to detect cellular and subcellular motion.

We thank Irving Itzkan and Lev Perelman of the MIT Spectroscopy Laboratory for valuable conversations and insights. This study was conducted at the MIT Laser Biomedical Research Center and was supported by grants from the National Institutes of Health (NIH). Adam Wax was supported by a National Research Service Award fellowship grant from the NIH.

\section{References}

1. P. J. Berne and R. Pecora, Dynamic Light Scattering (Wiley, New York, 1976).

2. R. Pecora, Dynamic Light Scattering: Applications of Photon Correlation Spectroscopy (Plenum, New York, 1985).

3. G. Maret and P. E. Wolf, "Multiple light scattering from dis- ordered media. The effect of Brownian motion of scatterers," Z. Phys. B 65, 409-413 (1987).

4. D. J. Pine, D. A. Weitz, P. M. Chaikin, and E. Herbolzheimer, "Diffusing-wave spectroscopy," Phys. Rev. Lett. 60, 1134-1137 (1988).

5. P.-A. Lemieux, M. U. Vera, and D. J. Durian, "Diffusing-light spectroscopies beyond the diffusion limit: the role of ballistic transport and anisotropic scattering," Phys. Rev. E 57, 44984515 (1998)

6. D. J. Durian, "Accuracy of diffusing-wave spectroscopy theories," Phys. Rev. E 51, 3350-3358 (1995).

7. M. Kempe, A. Z. Genack, W. R. Dorn, and P. Dorn, "Ballistic and diffuse light detection in confocal and heterodyne imaging systems," J. Opt. Soc. Am. A 14, 216-223 (1997).

8. M. J. Yadlowsky, J. M. Schmitt, and R. F. Bonner, "Multiple scattering in optical coherence microscopy," Appl. Opt. 34, 5699-5707 (1995).

9. K. K. Bizheva, A. M. Siegel, and D. A. Boas, "Path-lengthresolved dynamic light scattering in highly scattering random media: the transition to diffusing wave spectroscopy," Phys. Rev. E 58, 7664-7667 (1998).

10. D. A. Boas, K. K. Bizheva, and A. M. Siegel, "Using dynamic low-coherence interferometry to image Brownian motion within highly scattering media," Opt. Lett. 23, 319-321 (1998).

11. C. Scott, Introduction to Optics and Optical Imaging (Institute of Electrical and Electronic Engineers, Piscataway, N. J., 1998), p. 310.

12. A. G. Yodh, P. D. Kaplan, and D. J. Pine, "Pulsed diffusingwave spectroscopy: high resolution through nonlinear optical gating," Phys. Rev. B 42, 4744-4747 (1990).

13. R. Bonner and R. Nossal, "Model for laser Doppler measurements of blood flow in tissue," Appl. Opt. 20, 2097-2107 (1981).

14. A. H. Gandjbakhche, R. F. Bonner, and R. Nossal, "Scaling relationships for anisotropic random walks," J. Stat. Phys. 69, 35-53 (1992).

15. G. Yao and L. V. Wang, "Monte Carlo simulation of an optical coherence tomography signal in homogeneous turbid media," Phys. Med. Biol. 44, 2307-2320 (1999).

16. S. Fraden and G. Maret, "Multiple light scattering from concentrated, interacting suspensions," Phys. Rev. Lett. 65, 512515 (1990). 